\title{
Chlamydia trachomatis and reproductive health: what can we learn from systematic reviews of observational studies?
}

\author{
Nicola Low
}

\begin{abstract}
We are used to reading that Chlamydia trachomatis (CT) infection in pregnancy is associated with a range of adverse outcomes, including preterm birth, low birth weight, spontaneous abortion, intrauterine growth restriction and neonatal death. ${ }^{1}{ }^{2}$ These statements, often in the introduction to commentaries or research studies, are accompanied by references to general textbooks ${ }^{3}$ or studies in which strong associations have been found. ${ }^{4} 5$ But, how sure are we of these findings, why do we want to know and what can we learn?
\end{abstract}

Tang and colleagues have done a systematic review and meta-analysis, ${ }^{6}$ which substantially strengthen the completeness of the evidence base about the association between CT and adverse reproductive outcomes. They reviewed reports of 107 observational epidemiological studies (case-control, cohort or cross-sectional) published up to May 2018. Following good practice in systematic reviews of observational studies of aetiology, $^{7}$ they defined the research question in a published protocol, stratified results by study design, provided unadjusted and confounder-adjusted estimates where available, assessed the risk of bias and examined reasons for heterogeneity. The 107 studies in this review reported on any of 12 outcomes of pregnancy or fertility. Tang et al's extensive review is important for three main reasons. First, it is probably the largest systematic review of studies reporting on associations between C. trachomatis and reproductive outcomes. Second, the review finds empirical evidence that study setting, study design and analysis are associated with the strength of associations. Third, this review makes us think about what research studies are needed next.

In observational studies of aetiology, there are multiple sources of bias that affect their interpretation. In an earlier

Correspondence to Professor Nicola Low, Institute of Social and Preventive Medicine, University of Bern, Bern 3012, Switzerland; nicola.low@ispm.unibe.ch systematic review, Olson-Chen et al examined eight adverse outcomes of pregnancy, but not fertility, and included 56 studies published between 1970 and $2013,{ }^{8}$ with some overlap in outcomes and included studies with Tang et al. In both reviews, the strength of association was smaller in studies judged to be of better methodological quality. ${ }^{6} 8$ In neither review, however, did the authors identify which specific biases were associated with attenuation in the effect size. With so many outcomes and results, it can be hard to examine and discuss them all in detail. This commentary is a chance to examine, in a bit more detail, two methodological issues for systematic reviews and individual studies that could either overestimate or underestimate the strength of association between CT and adverse outcomes and to discuss implications for future research focusing on STIs in pregnancy.

Pregnancy is an interesting and special case for observational epidemiological studies. From beginning to end, it can be seen as a short cohort study. This short duration reduces some of the differences in the 'conventional' strengths and weaknesses between study designs. ${ }^{9}$ For example, in the assessment of causality, cross-sectional studies are usually seen as providing low certainty evidence, followed by case-control studies, with prospective cohort studies being the strongest observational design. ${ }^{10}$ This hierarchy may be true for chronic conditions and ongoing exposures because the temporal sequence of cause and effect cannot be determined and may be affected by recall bias in cross-sectional and some case-control studies. In cohort studies, the exposure status is defined first and its association with new, incident cases of the outcome determined during follow-up. If the study is examining outcomes of pregnancy, such as preterm delivery, the end of the pregnancy defines both the occurrence of the outcome and the end of a woman's involvement in the cohort study.

\section{CONFOUNDING}

Confounding is the major threat to the internal validity of observational epidemiological studies, so in a systematic review, the confounder-adjusted summary effect will provide the most useful information for questions about aetiology. ${ }^{7}$ CT and pregnancy are both sexually acquired, so many factors, including younger age, smoking, lower socioeconomic position and belonging to black or indigenous minority ethnic groups ${ }^{11}$ are associated with both and could plausibly result in systematic overestimation of exposureoutcome association. Tang et al chose to present unadjusted analyses as their primary outcome, because these were more often reported (178 effect estimates) than adjusted estimates (51 estimates). For outcomes with both univariable and multivariable analyses, the unadjusted estimate was higher than the adjusted estimate in 16 of 19 pairs, suggesting that confounding is present. For example, for preterm labour, the unadjusted and adjusted risk ratios in cohort studies were 1.54 (95\% CI 1.48 to 1.60$)$ and 1.09 (95\% CI 1.03 to 1.15$)$ and ORs in case-control studies were 1.29 (95\% CI 1.11 to 1.50$)$ and 1.15 (95\% CI 0.95 to 1.39 ), respectively. ${ }^{6}$

The presence of other genital tract infections, which are commonly found in women with CT and are also associated with specific outcomes, could also result in confounding. Tang et al did not report on coinfections and whether these had been measured or included in multivariable analyses. They did find that unadjusted effect estimates from case-control studies were higher in low-income and middle-income countries where, plausibly, women are both more likely to have had other genital tract infections associated with adverse pregnancy outcomes and might also have been less likely to have received treatment.

\section{MEASUREMENT BIAS}

The investigation of associations between curable infections and pregnancy outcomes brings special challenges because treatment, at an early enough stage, could reduce or eliminate the risk of the outcome. Indeed, that is usually the intention of treatment. Recording a woman as having CT when the infection was treated will result in mismeasurement of the exposure. In this situation, a cross-sectional study of the association between CT and adverse pregnancy outcomes could, paradoxically, provide a less biased effect estimate than a cohort or case-control study in which women were treated (Figure 1). 
Pregnancy

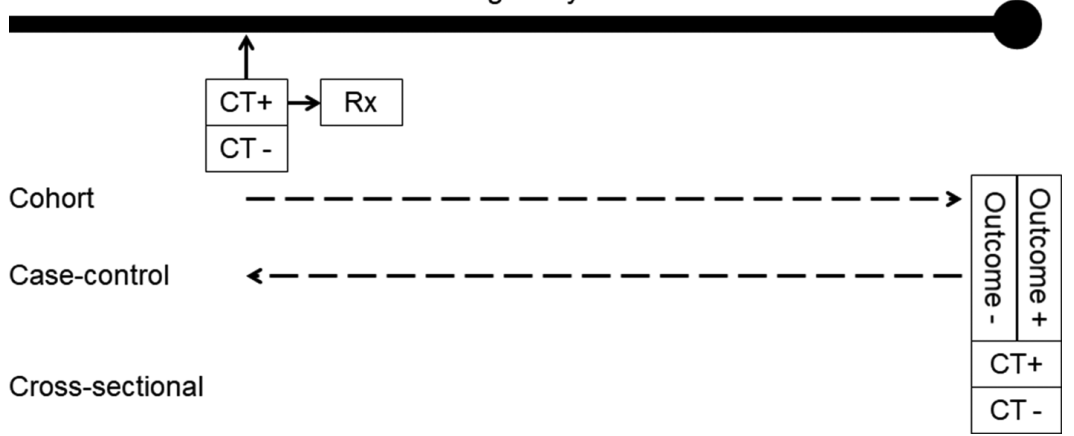

Figure 1 Observational epidemiological study designs to examine the association between CT and pregnancy outcomes, and the temporal relationship between detection of $\mathrm{CT}$, with Rx if CT+, and pregnancy outcome. The thick solid line indicates the course of pregnancy, with delivery at the end. In a cohort study, CT infection status is determined during pregnancy and, if present, is usually treated. The dashed line, with arrow pointing to the end of pregnancy, shows when the outcome is ascertained. In a case-control study, the dashed line starting at the end of pregnancy shows that the outcome is ascertained at delivery. The arrow points back in time to show that CT infection status is often determined through a lookback in medical records and, if detected, is likely to have been treated. In a cross-sectional study, both outcome and CT infection status are determined at the end of pregnancy and CTI infection is likely to be untreated. Abbreviations: CT, Chlamydia trachomatis; CT+, CT-infected; CT-, CT-uninfected; Rx, treatment.

In a cross-sectional study, a positive test result for CT taken at the time of delivery means that an untreated infection was present before or during pregnancy (or there is persistence/reinfection of a treated infection). The measured association is between untreated CT and pregnancy outcome (if adjusted for confounding). In cohort and case-control studies, CT tests are either done prospectively or records of tests done during pregnancy are sought. Women with CT diagnosed during pregnancy are likely to have been treated so the measured association is between treated CT and pregnancy outcomes. Tang et al found that summary estimates in metaanalyses of preterm labour and spontaneous abortion (but not low birth weight and premature rupture of membranes) were higher for cross-sectional than either cohort or case-control studies. There were no cross-sectional study details of other pregnancy outcomes, and Tang et al reported that details of CT testing and treatment were very rarely reported in the included studies.

\section{WHAT DO WE WANT TO KNOW?}

STI practitioners and researchers probably want to know whether CT infection in pregnancy is an important cause of poor reproductive health outcomes. Tang et al's review shows associations with several adverse outcomes. Because of the wide scope of the review, there was little opportunity to discuss the questions about causality or of potential mechanisms for individual outcomes. For example, the authors suggest that there is increased evidence of associations with stillbirth and spontaneous abortion. ${ }^{6}$ However, these associations were only present in unadjusted analyses, the biological mechanism is unclear, and the certainty of the body of evidence was assessed as low or very low.

The systematic review by Tang et al does not allow a precise quantification of the causal association between CT infection and the multiple potential adverse outcomes for women's reproductive health. Their findings will, however, be relevant to the interpretation of systematic reviews of associations between other sexually transmitted and genital tract infections and pregnancy outcomes. ${ }^{8} 12 \quad 13$ The review also highlights methodological issues that researchers can take into account for future studies. Individual studies of associations between genital tract infections and pregnancy outcomes should examine a full range of potential pathogens, including the vaginal microbiota, record the timing of testing and treatment for infection, and both measure and control for confounding factors. Improved reporting of observational studies ${ }^{14}$ will make it easier for results to be incorporated in systematic reviews. ${ }^{7}$ Doing a systematic review requires more than following a recipe. In systematic reviews of observational studies, authors should consider in advance what are the important confounding factors for each exposure-outcome pair, show results of both unadjusted and confounder-adjusted estimates, and assess the risk of bias considering that sources of bias may be specific to different research questions and study designs.
Improved reproductive health outcomes are a major international goal for women and their families, for clinical medicine and for public health. Systematic reviews can help to investigate the potential population impact of interventions. The studies identified and included will point to gaps in the evidence, and summary effect sizes can be used to estimate population-attributable fractions (bearing in mind the strong assumptions about causality). Ultimately, randomised controlled trials of different interventions, with clinical outcomes, in settings with low and high levels of CT and coinfection prevalence will be needed to determine whether screening and treatment to prevent adverse pregnancy outcomes do more good than harm at a reasonable cost.

\section{Handling editor Anna Maria Geretti Twitter Nicola Low @nicolamlow}

Contributors NL conceived and wrote the editorial.

Funding The authors have not declared a specific grant for this research from any funding agency in the public, commercial or not-for-profit sectors.

Competing interests NL is a deputy editor of Sexually Transmitted Infections. She is also involved in systematic reviews and randomised controlled trials about sexually transmitted infections in pregnancy.

Patient consent for publication Not required.

Provenance and peer review Commissioned; internally peer reviewed.

(C) Author(s) (or their employer(s)) 2020. No commercial re-use. See rights and permissions. Published by BMJ.

\section{Check for updates}

To cite Low N. Sex Transm Infect 2020;96:315-317. Received 18 December 2019

Accepted 10 January 2020

Published Online First 27 January 2020

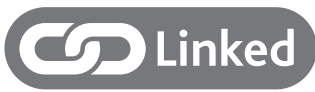

- http://dx.doi.org/10.1136/sextrans-2019-053999

Sex Transm Infect 2020;96:315-317.

doi:10.1136/sextrans-2019-054279

ORCID iD

Nicola Low http://orcid.org/0000-0003-4817-8986

\section{REFERENCES}

1 Blas MM, Canchihuaman FA, Alva IE, et al. Pregnancy outcomes in women infected with Chlamydia trachomatis: a population-based cohort study in Washington state. Sex Transm Infect 2007:83:314-8.

2 Rours GIJG, Duijts L, Moll HA, et al. Chlamydia trachomatis infection during pregnancy associated with preterm delivery: a population-based prospective cohort study. Eur J Epidemiol 2011;26:493-502.

3 Holmes KK, Sparling PF, Stamm WE, et al. Sexually transmitted diseases. New York: McGraw-Hill, 2008

4 Andrews WW, Goldenberg RL, Mercer B, et al. The preterm prediction study: association of secondtrimester genitourinary Chlamydia infection with 
subsequent spontaneous preterm birth. Am J Obstet Gynecol 2000;183:662-8.

5 Gravett MG, Nelson HP, DeRouen T, et al. Independent associations of bacterial vaginosis and Chlamydia trachomatis infection with adverse pregnancy outcome. JAMA 1986;256:1899-903.

6 Tang W, Mao J, Li KT, et al. Pregnancy and fertility-related adverse outcomes associated with Chlamydia trachomatis infection: a global systematic review and meta-analysis. Sex Transm Infect 2020;96:322-9.

7 Dekkers OM, Vandenbroucke JP, Cevallos M, et al. COSMOS-E: guidance on conducting systematic reviews and meta-analyses of observational studies of etiology. PLoS Med 2019;16:e1002742.
8 Olson-Chen C, Balaram K, Hackney DN. Chlamydia trachomatis and adverse pregnancy outcomes: metaanalysis of patients with and without infection. Matern Child Health I 2018:22:812-21.

9 Wilcox AJ. Reproductive epidemiology: themes and variations. In: Fertility and pregnancy an epidemiologic perspective. Oxford: Oxford University Press, 2010: 115-20.

10 Vandenbroucke JP. Observational research, randomised trials, and two views of medical science. PLoS Med 2008:5:e67

11 Liu B, Roberts CL, Clarke M, et al. Chlamydia and gonorrhoea infections and the risk of adverse obstetric outcomes: a retrospective cohort study. Sex Transm Infect 2013:89:672-8.
12 Vallely LM, Egli-Gany D, Pomat W, et al. Adverse pregnancy and neonatal outcomes associated with Neisseria gonorrhoeae, Mycoplasma genitalium, M. hominis, Ureaplasma urealyticum and U. parvum: a systematic review and meta-analysis protocol. BMJ Open 2018;8:e024175.

13 Silver BJ, Guy RJ, Kaldor JM, et al. Trichomonas vaginalis as a cause of perinatal morbidity: a systematic review and meta-analysis. Sex Transm Dis 2014;41:369-76.

14 von Elm E, Altman DG, Egger M, et al. The strengthening the reporting of observational studies in epidemiology (STROBE) statement: guidelines for reporting observational studies. PLOS Med 2007;4:e296 